\title{
Validation of a Radial Compressor CFD Analysis
}

\author{
${ }^{1 *}$ Suleyman Emre Ak and ${ }^{1}$ Sertac Cadirci \\ ${ }^{1}$ Department of Mechanical Engineering, Istanbul Technical University, 34437, Istanbul, Turkey
}

\begin{abstract}
In the present study, compressible high speed flow in a radial compressor is investigated by Computational Fluid Dynamics (CFD) and the numerical results of pressure ratios and adiabatic efficiencies are validated against available data in the literature. The radial compressor of interest operates at a rotational speed of $21789 \mathrm{rpm}$ and its main components are rotor, diffuser and exit guide vanes. The main motivation of the study is to determine the appropriate CFD approach and boundary conditions of the problem that will fit best to the measurements. The entire maps of the three-stage radial compressor have been obtained by CFD analysis and they agreed well with the measurements with maximum relative errors of approximately $3.6 \%$ and $1.3 \%$ for the adiabatic efficiency and the compression ratio, respectively
\end{abstract}

Key words: High Efficiency Centrifugal Compressor (HECC), Compressible Flow, Computational Fluid Dynamics (CFD).

\section{Introduction}

In literature there are many numerical studies to simulate compressible flow in radial compressors. In the studies [1-5] the researchers investigated different CFD modeling settings about interfaces and boundary conditions to successfully model compressible flow in various types of high speed compressors. Liu and Hill [5] discussed different interface models that can be implemented to rotational and stationary parts of the turbo-compressor and pointed out that the results from circumferential averaging type interface approach were more similar to transient analysis than the frozen rotor interface for a vaned diffuser radial compressor. The study of Gibson et. al. [7] provided a detailed comparison of turbulence models for a two-stage radial compressor with vaned diffuser. They compared five different turbulence models and recommended the k- $\omega$ SST turbulence model [10] for the entire speed line of the compressor. In many related studies, $k-\omega$ SST turbulence model proposed by Menter was used by many researchers in their CFD simulations for compressible flows in radial compressors $[2,6,8,9]$.

In the present study, a benchmark problem was utilized to validate the CFD results of the radial compressor of interest. The measurements of the High Efficiency Centrifugal Compressor (HECC) used in the present study for validation are taken from the experiments which have been conducted in National Aeronautics and Space Administration (NASA) Small Engine Components Test Facility. In these experiments, HECC impeller was operated at five different speeds in the range between surge and choke lines. The geometry of the HECC, the data of the measurements and the measurement instrumentations used in the test rig are available in the NASA contractor report 'High Efficiency Centrifugal Compressor for Rotorcraft Applications (2014)' [11].

*Corresponding author: Suleyman Emre Ak, Address: Department of Mechanical Engineering, Istanbul Technical University, 34437 Istanbul TURKEY. E-mail address: ak@itu.edu.tr, Phone: +90 2122931300 


\section{HECC Geometry}

HECC is an open-source geometry designed by NASA and United Technologies Research Center (UTRC). HECC geometry can be generated from hub, shroud and blade profile coordinates, its CAD model and meridional section are shown in Fig. 1. The main parts of the HECC geometry are a set of three blade rows figuring out the impeller, diffuser and the exit guide vane (EGV). In this configuration, the impeller is composed of fifteen pairs of main and splitter blades, the diffuser row is composed of twenty pairs of main and splitter blades and EGV has sixty cascade type blades. Impeller blades are backswept and have a $0.3 \mathrm{~mm}$ tip clearance. All the blades have elliptical leading and trailing edges.

The computational domain consists of the rotating impeller, stationary diffuser and EGV. In the CFD analysis for both the impeller and the diffuser one main and one splitter blade are selected and the EGV is represented by only one blade. As a result of this path selection from inlet to outlet in the computational domain, the angles for the impeller, diffuser and the EGV are $24^{\circ}, 18^{\circ}$ and $6^{\circ}$, respectively. Thus, the pitch ratio between these three domains is not equal which requires the usage of the mixing-plane interface between the domains.
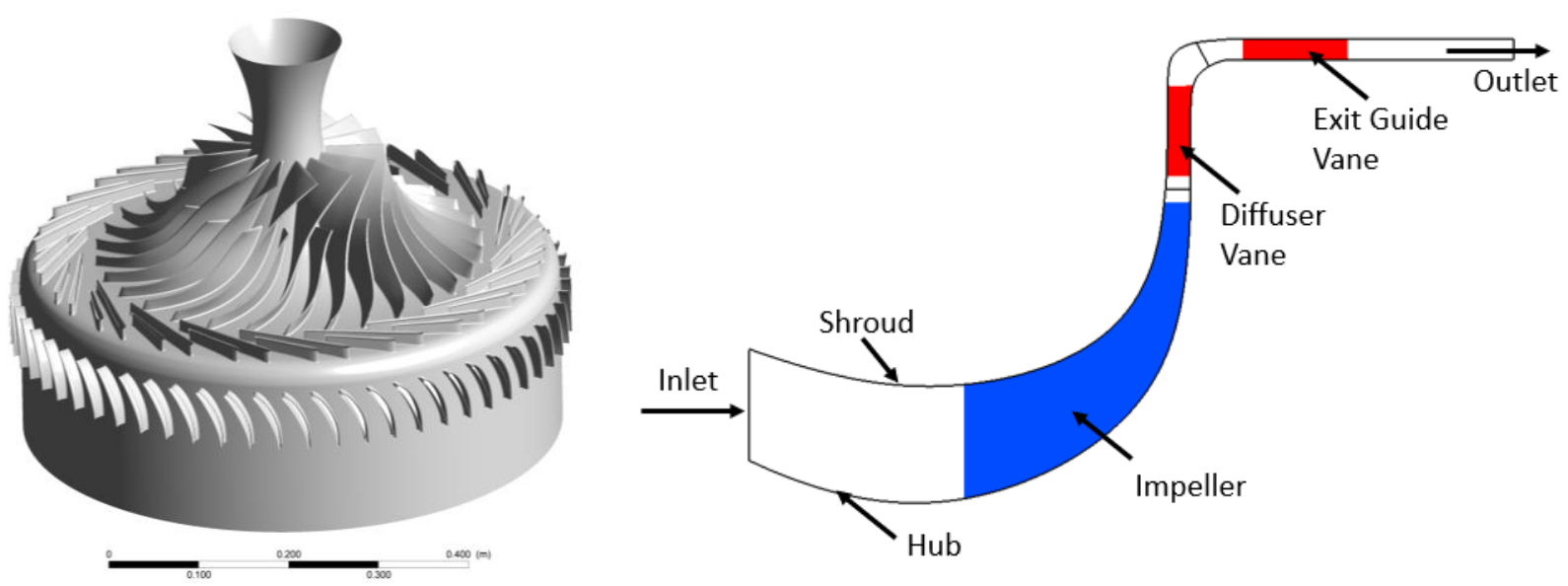

Figure 1. CAD model (left) and the meridional section of the HECC (right).

\section{Numerical Modeling}

In this section the main features of the computational domain with the boundary conditions and mesh convergence tests are presented.

\subsection{CFD Approach and Boundary Conditions}

At the inlet of the radial compressor the total pressure of $101325 \mathrm{~Pa}$ and the total temperature of $15^{\circ} \mathrm{C}$ are defined for air at a design mass flow rate of $5.11 \mathrm{~kg} / \mathrm{s}$. The design rotational speed of the impeller is $21789 \mathrm{rpm}$, in the present study $\% 100$ and $90 \%$ of this design speed are used. It should be noted that the shroud wall of the impeller domain is non-rotating. For other operating conditions, 
different mass flow rates are defined as outlet boundary condition. CFD studies have been carried out using flow solver software ANSYS-CFX version 2020 R1. In the CFD calculations, steadystate compressible flow is modelled using ideal gas approach for air. One blade from each blade set is used to decrease computational cost and save computational time. Circumferential averaging type interface is used between domains. As suggested in literature, k- $\omega$ SST turbulence model is selected since it can predict the flow variables close to measurements with relatively low computational effort.

\subsection{Mesh Sensitivity}

After extensive mesh sensitivity tests the adequate mesh with sufficient number of elements and necessary mesh requirements is determined as shown in Table 1. Mesh sensitivity tests are carried out with the design boundary and operating conditions, namely $5.11 \mathrm{~kg} / \mathrm{s}$ mass flow rate and 21789 rpm impeller speed. Hexahedral elements are used in all domains. A mesh with nearly 11.3 million cells is found to be sufficient and decided for further calculations. Figure 2 shows the representative mesh topology in the vicinity of the leading edge of the impeller.

Table 1. Mesh Sensitivity Tests

\begin{tabular}{ccccccc}
\hline & $\begin{array}{c}\text { Number of } \\
\text { Cells } \\
{[\text { millions }]}\end{array}$ & $\begin{array}{c}\text { Adiabatic } \\
\text { Efficiency } \\
{[\%]}\end{array}$ & $\begin{array}{c}\text { Relative } \\
\text { Error for } \\
\text { Adiabatic } \\
\text { Efficiency } \\
{[\%]}\end{array}$ & $\begin{array}{c}\text { Pressure } \\
\text { Ratio } \\
{[-]}\end{array}$ & $\begin{array}{c}\text { Relative } \\
\text { Error for } \\
\text { Pressure } \\
\text { Ratio } \\
{[\%]}\end{array}$ & $\begin{array}{c}\text { Average } \\
\mathrm{y}^{+} \\
{[-]}\end{array}$ \\
\hline Experiment & - & 80.31 & - & 4.506 & - & - \\
Mesh 1 & 4.7 & 82.05 & 2.17 & 4.458 & 1.06 & 9.00 \\
Mesh 2 & 8.0 & 82.70 & 2.96 & 4.521 & 0.33 & 5.87 \\
Mesh 3 & 11.3 & 82.89 & 3.20 & 4.544 & 0.84 & 2.62 \\
Mesh 4 & 18 & 82.99 & 3.29 & 4.550 & 0.97 & 1.84 \\
Mesh 5 & 25 & 82.99 & 3.33 & 4.553 & 1.03 & 1.45 \\
\hline
\end{tabular}

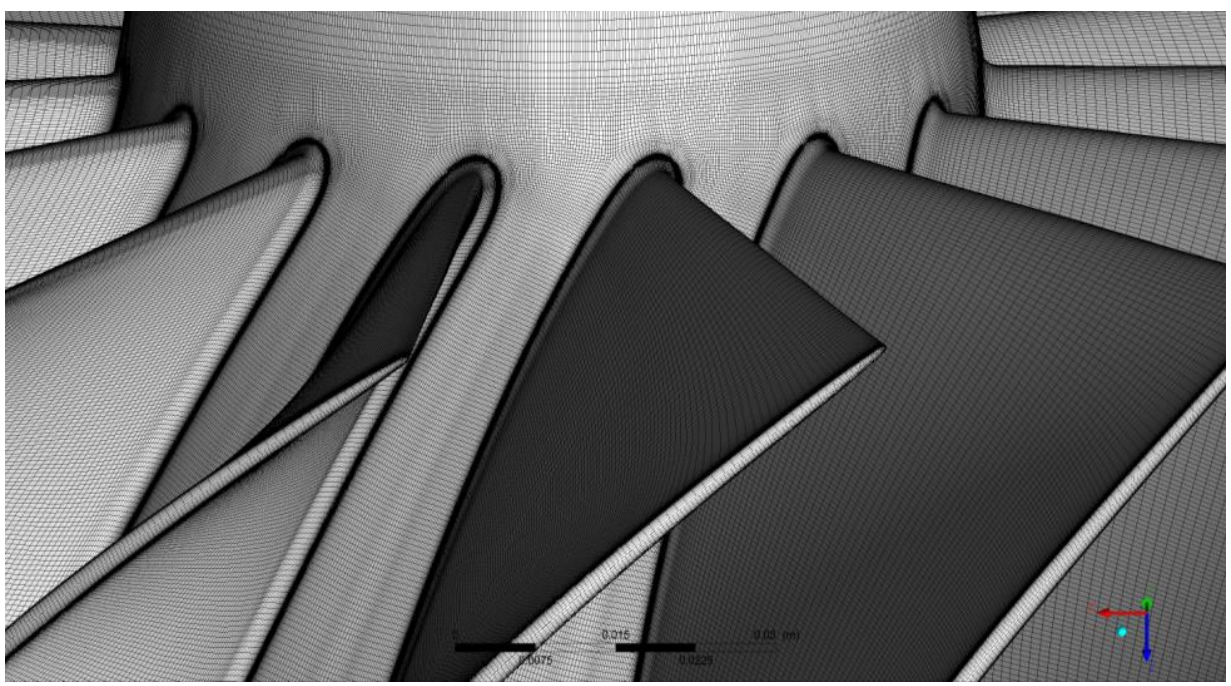

Figure 2. A representative mesh topology 


\section{Results}

In the CFD calculations the entire compressor map for pressure ratios and adiabatic efficiencies are plotted against all operating points given in the experiments as demonstrated in Fig. 3a, and 3b, respectively. It is shown that there is an acceptable and convincing agreement between the predicted CFD results and the measurements. The main reason for the deviations at highest mass flow rates is 'choking' which should be linked to the mass flow rate boundary condition at the outlet. In experimentations the real boundary condition at the outlet is pressure outlet. But this phenomenon is insignificant in HECC mapping since the study focuses on the surge region.
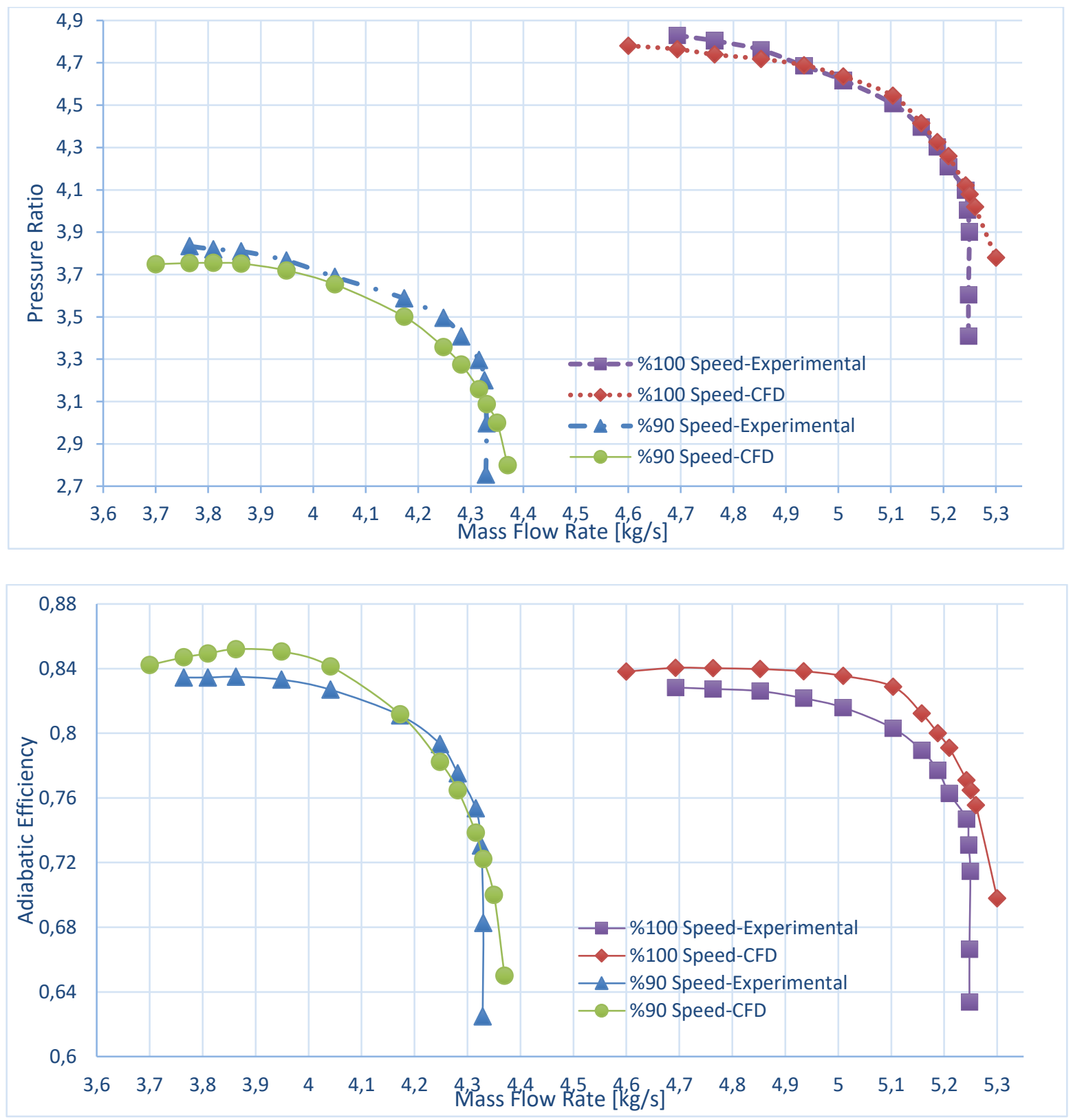

Figure 3. Maps for (a) pressure ratio, (b) adiabatic efficiency at design (100\%) and 90\% speeds. 
Fig. 4 indicates the flow fields in the diffuser and EGV sections in color scales compared between design $(5.11 \mathrm{~kg} / \mathrm{s})$ and surge mass flow rates $(4.7 \mathrm{~kg} / \mathrm{s}$, lowest mass flow rate) at the design rotational speed (100\%). If the compressor is operated at the design mass flow rate, a notable flow separation zone occurs on main diffuser vane's pressure surface near the leading edge. If operated at a surge mass flow rate, the flow separation zone forms on the suction surface of diffuser vane close to the trailing edge as demonstrated in Fig.5. However, flow separation is observed on the suction surface of the EGV blades at both design and surge mass flow rates.
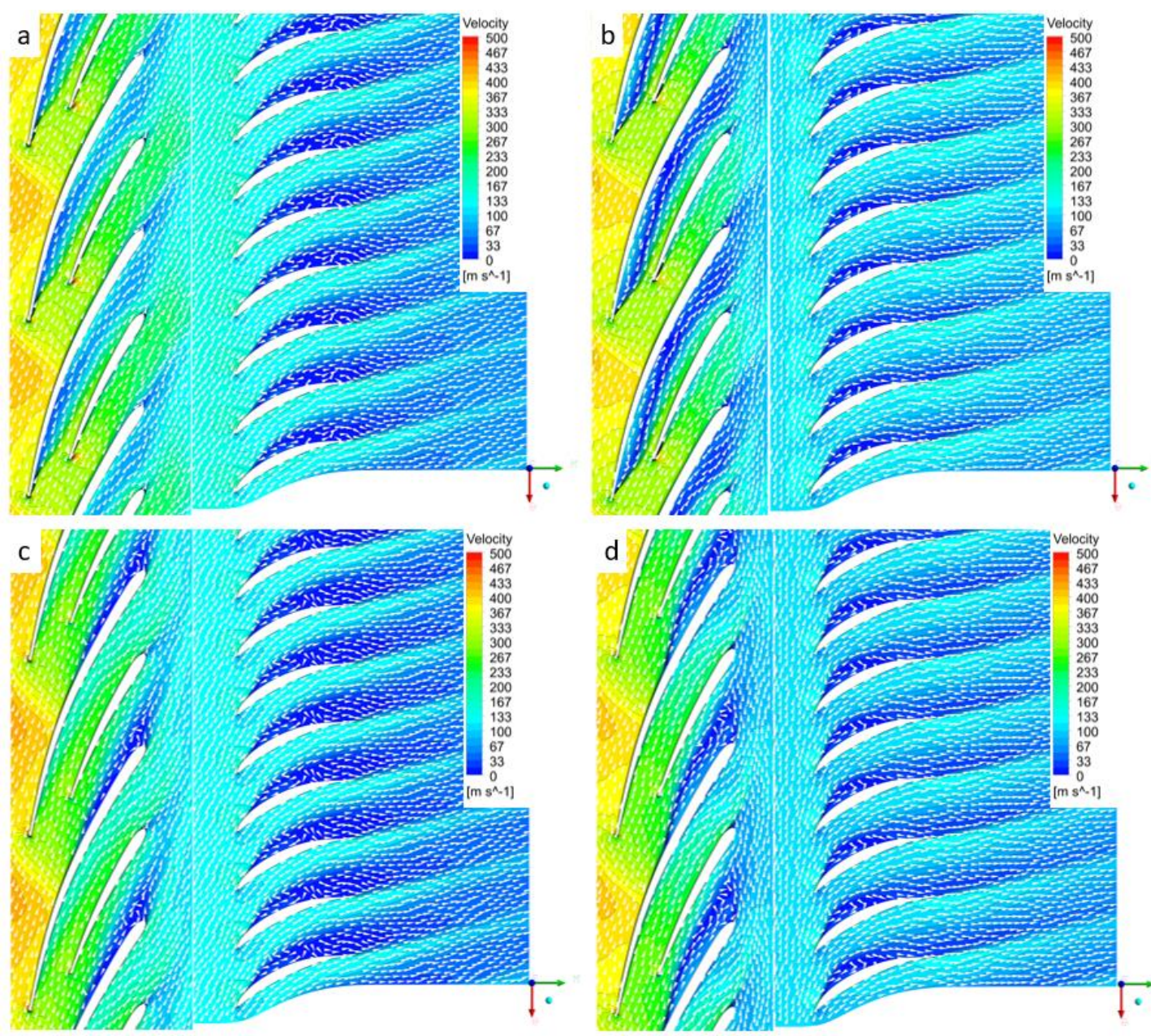

Figure 4. Flow fields in diffuser and EGV domains: Design mass flow rate at (a) $50 \%$ span, (b) $25 \%$ span and Surge mass flow rate at (c) $50 \%$ span, (d) $25 \%$ span (View is duplicated).

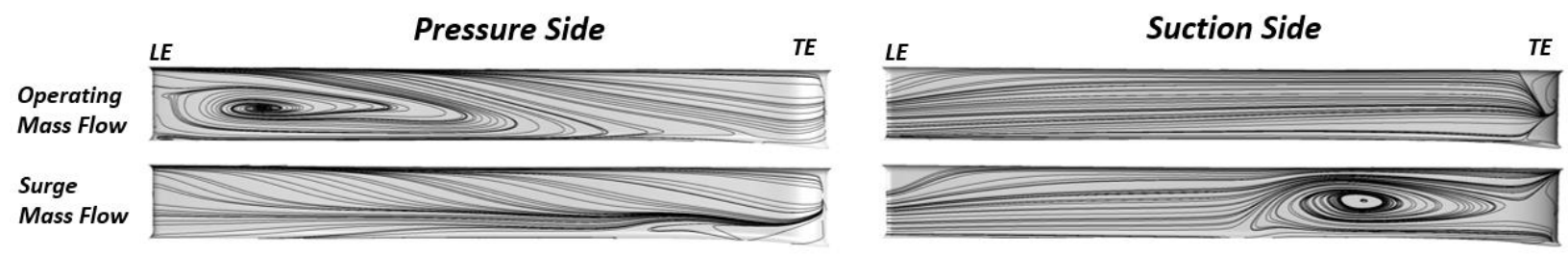

Figure 5. Surface streamlines indicating flow separation zones on diffuser vane surfaces. 
The CFD analysis reveal that flow separation zones occur at different locations on the diffuser blades at various span-wise sections depending on the mass flow rates. This suggests that the appropriate choice of the mass flow rate is vital in understanding flow separation on the diffuser blades' surfaces.

Figure 6 shows the pressure contours at $50 \%$ span of the radial compressor at the design mass flow rate and the surge mass flow rate. In the impeller part, the pressure distributions are almost identical for both mass flow rates. However, the most notable difference in the pressure contours is observed in the diffuser section. If the radial compressor is operated at a surge mass flow rate, the pressure gradient at the leading edge of the main diffuser blades are higher than the design mass flow rate. This suggests that the appropriate choice of the mass flow rate is also important in the magnitude of the pressure between the impeller and the diffuser.

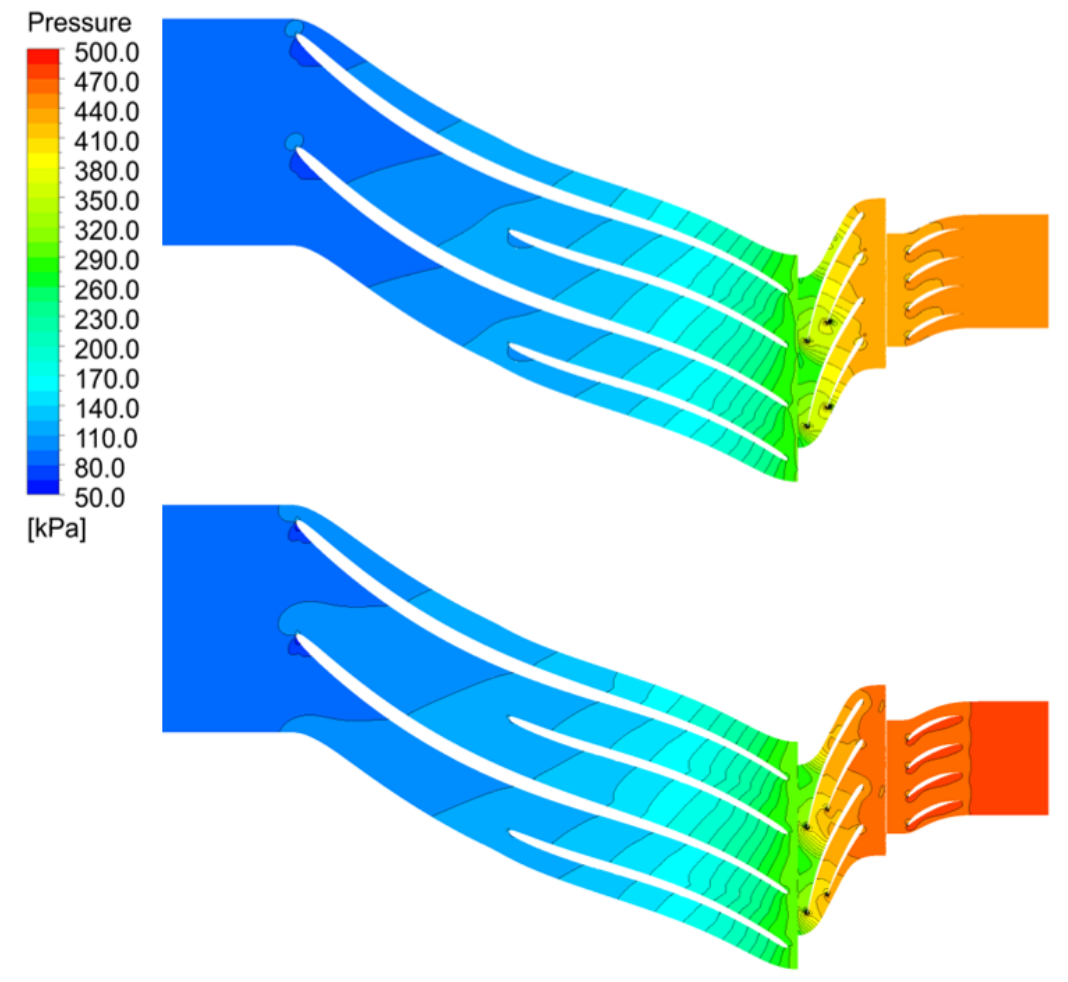

Figure 6. Pressure distribution at 50\% span: Design mass flow rate (Top), Surge mass flow rate (Bottom) (View is duplicated).

\section{Conclusions}

In this study, CFD analysis have been carried out for a radial compressor known as HECC in literature and the results are compared against the reported measurements. The analysis are conducted using $k-\omega$ SST turbulence model for steady compressible air flow with ideal-gas assumption as proposed in literature. All the operating points (13-14 points) have been taken into consideration and a convincing agreement is found between numerical and experimental data in the entire operation range between surge and choke lines where the maximum relative errors are 
$3.6 \%$ and $1.3 \%$ for the adiabatic efficiency and the pressure ratio, respectively. Choking could not be successfully modelled because the outlet is subjected to mass flow rate boundary condition in the CFD analysis. The CFD analysis provide a deeper insight into the steady compressible flow and assist to better understand flow structures such as primary flow separation zones on the diffuser blades. Future studies can include active flow control strategies which can be an alternative solution to prevent or delay flow separation.

\section{References}

[1] Zheng XQ, Huenteler J, Yang MY, Zhang YJ, Bamba T. Influence of the volute on the flow in a centrifugal compressor of a high-pressure ratio turbocharger. Proceedings of the Institution of Mechanical Engineers, Part A: Journal of Power and Energy, vol. 224, no. 8, pp. 1157-1169, 2010.

[2] Rinaldi E, Pecnik R, Colonna P. Computational fluid dynamic simulation of a supercritical $\mathrm{CO} 2$ compressor performance map. Journal of Engineering for Gas Turbines and Power, vol. 137, Article ID 072602, 2015.

[3] Li J, Yin Y, Li S, Zhang J. Numerical simulation investigation on centrifugal compressor performance of turbocharger. Journal of Mechanical Science and Technology, vol. 27, no. 6, pp.1597-1601, 2013.

[4] Schreiber J, Ottavy X, Ngo Boum G, Aubert S, Sicot F. Numerical simulation of the flow field in a high speed multistage compressor: study of the time discretization sensitivity. Proceedings of the ASME Turbo Expo 2015: Turbine Technical Conference and Exposition, p. V02BT39A006, Montreal, Quebec, Canada, 2015.

[5] Liu Z, Hill D. Issues surrounding multiple frames of reference models for turbo compressor applications. Proceedings of the International Compressor Engineering Conference, 2000.

[6] Zamiri A, Lee BJ, Taek Chung J. Numerical evaluation of transient flow characteristics in a transonic centrifugal compressor with vaned diffuser. Aerospace Science and Technology,vol. 70, pp. 244-256, 2017.

[7] Gibson L, Galloway L, Kim SI, Spence S. Assessment of turbulence model predictions for a centrifugal compressor simulation. Journal of the Global Power and Propulsion Society, vol. 1, pp. 142-256, 2017.

[8] Anish S, Sitaram N. Computational investigation of impeller-diffuser interaction in a centrifugal compressor with different types of diffusers. Proceedings of the Institution of Mechanical Engineers, Part A: Journal of Power and Energy, vol. 223, no. 2, pp. 167-178, 2008.

[9] Roytta P, Grönman A, Jaatinen A, Turunen-Saaresti T, Backman J. Effects of different blade angle distributions on centrifugal compressor performance. International Journal of Rotating Machinery, vol. 2009, Article ID 537802, 2009.

[10] Menter F R. Two-Equation Eddy-Viscosity Turbulence Models for Engineering Applications. AIAA Journal, 32(8), pp. 1598-1605, 1994.

[11] Medic G, Sharma OP, Jongwook J, Hardin LW, McCormick DC, Cousins WT et al. High Efficiency Centrifugal Compressor for Rotorcraft Applications. NASA Contractor Report, NASA/CR-2014-218114, 2014. 